\title{
Postoperative mortality after inpatient surgery: Incidence and risk factors
}

\author{
Karamarie Fecho' \\ Anne T Lunney' \\ Philip G Boysen' \\ Peter Rock ${ }^{2}$ \\ Edward A Norfleet' \\ 'Department of Anesthesiology, \\ School of Medicine, University \\ of North Carolina, Chapel Hill, NC, \\ USA; ${ }^{2}$ Department of Anesthesiology, \\ University of Maryland, Baltimore, \\ MD, USA
}

\begin{abstract}
Purpose: This study determined the incidence of and identified risk factors for 48 hour (h) and 30 day (d) postoperative mortality after inpatient operations.

Methods: A retrospective cohort study was conducted using Anesthesiology's Quality Indicator database as the main data source. The database was queried for data related to the surgical procedure, anesthetic care, perioperative adverse events, and birth/death/operation dates. The $48 \mathrm{~h}$ and $30 \mathrm{~d}$ cumulative incidence of postoperative mortality was calculated and data were analyzed using Chi-square or Fisher's exact test and generalized estimating equations.

Results: The $48 \mathrm{~h}$ and $30 \mathrm{~d}$ incidence of postoperative mortality was $0.57 \%$ and $2.1 \%$, respectively. Higher American Society of Anesthesiologists physical status scores, extremes of age, emergencies, perioperative adverse events and postoperative Intensive Care Unit admission were identified as risk factors. The use of monitored anesthesia care or general anesthesia versus regional or combined anesthesia was a risk factor for $30 \mathrm{~d}$ postoperative mortality only. Time under anesthesia care, perioperative hypothermia, trauma, deliberate hypotension and invasive monitoring via arterial, pulmonary artery or cardiovascular catheters were not identified as risk factors
\end{abstract}

Conclusions: Our findings can be used to track postoperative mortality rates and to test preventative interventions at our institution and elsewhere.

Keywords: postoperative mortality, risk factors, operations, anesthesia, inpatient surgery

\section{Introduction}

Increased attention has been placed in recent years on improving quality and safety in healthcare. In the United States, the Institute of Medicine (IOM), the Joint Commission on the Accreditation of Healthcare Organizations (JCAHO), and the Institute for Healthcare Improvement (IHI) have made improvements in medication safety and adverse event tracking top priorities for the national public health agenda of the 21st century (IOM 2001; IHI 2007; JC 2007). The emphasis on patient safety was brought to the forefront by a 1999 report by the IOM which presented statistics suggesting that medical error accounts for the death of nearly 100,000 Americans each year (IOM 1999). That same report noted the field of anesthesiology as a model for patient safety. Indeed, in the early 1980s, rapid improvements in anesthesia safety were made over a very short period of time, primarily through the implementation of new clinical practice guidelines, improvements in vital signs monitoring technology, and the formation of the Anesthesia Patient Safety Foundation (IOM 1999; 2001; IHI 2007; JC 2007).

Yet, as emphasized by Lagasse (2002a; 2002b), claims that anesthesia remains safe today are based more on perception than on valid published data, as estimates on the incidence of anesthesia-related perioperative mortality are few, particularly in the United States. The most commonly cited report, from 1989, indicates that the rate of perioperative death directly attributable to anesthesia management is approximately $0.001 \%$ for generally healthy persons (Eichhorn 1989). Even less is known about longer 
term postoperative mortality, although a 2005 study by Monk and colleagues (2005) reports a one-year mortality rate of $5.5 \%$ after noncardiac surgery in adults. The perceived safety of anesthesia has encouraged more complex surgeries to be performed on patients who might have been considered too unhealthy for surgery in the recent past, and yet even proponents for the safety of anesthesia admit that further progress is needed to improve anesthesia safety for less healthy persons (Cooper and Gaba 2002). The perceived safety of anesthesia, coupled with economic pressures, also have contributed to the rise in the number of outpatient surgeries being conducted. Nonetheless, only two published studies, one by our group (Fecho et al in press) and one by another group (Warner et al 1993), focus on anesthesia-related adverse events in the outpatient setting.

The overall goals of our study were to determine the 48 hour (h) and 30 day (d) incidence of postoperative mortality at our university-based, tertiary care hospital and to identify risk factors for postoperative mortality. The potential risk factors we evaluated were patient age and American Society of Anesthesiologists (ASA) physical status (PS), type of anesthesia (monitored anesthesia care (MAC), general, regional or combined), time under anesthesia care, emergency or trauma status, the occurrence of a perioperative adverse event, perioperative hypothermia, deliberate intraoperative hypotension, the need for invasive monitoring (via arterial line, cardiovascular catheter or pulmonary artery), and postoperative intensive care unit (ICU) admission. Several of these factors have been implicated in earlier studies on anesthesia-related perioperative adverse events (Beecher and Todd 1954; Dripps et al 1961; Vacanti et al 1970; Eger et al 1971; Farrow et al 1982; Tiret et al 1986; Forrest et al 1992; Schwilk et al 1998), but have not been fully evaluated in recent years, and so the contribution of these factors to postoperative mortality in today's operating room environment remains unknown.

\section{Methods}

All study procedures were approved by our Institutional Review Board.

\section{Data sources}

Our Department of Anesthesiology's Quality Indicator (QI) database and the hospital's Surgical Services databases were the data sources. The QI database was maintained on a Microsoft SQL Server installed on a server running the Microsoft Windows 2000 Operating System (Microsoft Corp., Redmond, WA) and it contained data on every anesthesia-assisted procedure conducted at our hospital. The data were derived from a standard QI form similar to the one developed and validated by Cooper and colleagues (1987) for perioperative adverse event reporting. The form contained check-boxes for the occurrence of 48 specific types of perioperative adverse events in several major categories (Airway, Cardiovascular, Neurological, Regional, Respiratory and Miscellaneous-System Errors, Patient Factors, Other), and anesthetists completed the form in the postanesthesia care unit. The anesthesia record was used to obtain additional data that also were entered into the database: the patient's date of birth and ASA PS; type of anesthesia; date and time that anesthesia care began and ended; emergency or trauma status of the operation; perioperative hypothermia; deliberate intraoperative hypotension; the need for invasive monitoring via arterial line, cardiovascular catheter or pulmonary artery catheter; and whether the patient was admitted (planned or unplanned) to the ICU postoperatively. The hospital's Surgical Services database was used to obtain data on the date of death for any patient who dies after admission. Anesthesiology's QI database was updated with a patient's date of death on a daily basis. For the purpose of the present study, we queried the QI database for all fields of data for each procedure conducted between 4/1/04 and 3/31/05. The data were successfully extracted and downloaded into a Microsoft Office Excel 2003 spreadsheet for transfer. The dataset was then uploaded into SPSS (version 15.0 for Windows; SPSS Inc., Chicago, IL) for variable "clean-up," variable building and variable transformation prior to statistical analysis.

\section{Endpoint variables}

The time to death was calculated as the number of days from the patient's date of surgery to the date of death (rounded to the nearest day). Mortality variables were created for two time periods after surgery, $48 \mathrm{~h}$ or $30 \mathrm{~d}$, and mortality was treated as a binary variable ( 0 if did not die or 1 if died) for each time period.

\section{Risk factors}

The standard ASA PS scale of 1-5 was used in this study; procedures classified as ASA PS 6 (organ donor) were excluded from the dataset prior to analysis (Vacanti et al 1970). Patient age was calculated as the number of days from the patient's date of birth to the date of the procedure, and age was expressed as years (rounded to the nearest year). Chronological age was used to calculate the age of premature babies. Patient age was categorized as $0-1,2-17,18-39$, 40-64, and $65+$ years, following the age categories used by 
Monk and colleagues (2005). Four types of anesthesia were evaluated: MAC; regional anesthesia; general anesthesia; or a combination. Total time under anesthesia care was calculated as the number of minutes (rounded to the nearest minute) between the date and time that anesthesia care was begun to the date and time that anesthesia care ended. Total time under anesthesia care was broken into quartiles for analysis, which corresponded roughly to operation times of $<60 \mathrm{~min}$ (quartile 1), 60-120 min (quartile 2), 120-240 min (quartile 3), and $>240 \mathrm{~min}$ (quartile 4). The emergency or trauma status of the operation, perioperative hypothermia, deliberate intraoperative hypotension and postoperative ICU admission were classified as binary variables ( 0 if no, 1 if yes). The total number of perioperative adverse events associated with each operation was calculated and a new variable was created to treat the occurrence of adverse events as a binary variable ( 0 or $\geq 1$ adverse events). We did not evaluate subcategories of perioperative adverse events as the numbers within each subcategory were quite small, but of the 290 adverse events that were reported, the most common events were cardiovascular (55 or 19.1\%) and respiratory (54 or $18.6 \%$ ). Only inpatient operations were analyzed; outpatient and nonsurgical procedures (eg, electroconvulsive therapy) were excluded prior to analysis.

\section{Statistical analysis}

The data were analyzed using SPSS (SPSS Inc.) and graphed using Sigma Plot 2000 (version 6.1; SPSS Inc.). We treated each instance of death as an individual endpoint variable (coded as a binary variable, 0 or 1 ). Cumulative incidence rates of mortality and crude mortality rates were calculated for $48 \mathrm{~h}$ and $30 \mathrm{~d}$ postoperatively. The cumulative incidence of mortality was calculated for each level of the evaluated risk factors. Univariate analyses using chi-square or Fisher's exact test were used to compare mortality rates across potential risk factors, with $\alpha=0.05$. Each risk factor was recategorized as a binary variable (if not already) for multivariate analysis using a generalized estimating equations model to determine the strength of each factor as a predictor of postoperative mortality and to estimate odds ratios, or relative risks. We categorized in this manner because, conceptually, the odds ratio is easier to understand and interpret when applied to binary variables. Subject identifier was included as a covariate in the generalized estimating equations model to control for subjects having $>1$ operation over the sampling period, as those subjects may be assumed to have greater comorbidity. For all analyses, $\mathrm{p}<0.05$ was considered significant.

\section{Results}

From 4/1/04 through 3/31/05, 12,739 inpatient operations were conducted at our hospital. The incidence of postoperative hospital mortality was $0.57 \%$ within $48 \mathrm{~h}$ and $2.1 \%$ within $30 \mathrm{~d}$ of an inpatient operation (Table 1). These rates translate into crude $48 \mathrm{~h}$ mortality rates of 1:177 operations and crude $30 \mathrm{~d}$ mortality rates of 1:49 operations.

Our univariate analyses showed that the incidence of mortality varied across evaluated risk factors (Figures 1 and 2). Mortality rates $48 \mathrm{~h}$ and $30 \mathrm{~d}$ postoperatively increased with increased ASA PS ( $p<0.0005)$. The same relationship was found even when ASA 5 patients were excluded from analysis $(\mathrm{p}<0.0005)$. Mortality rates were higher for very young $(0-1$ years $)$ and older $(65+$ years $)$ patients $(\mathrm{p}<0.0005)$. Emergency or trauma operations were associated with higher $48 \mathrm{~h}$ and $30 \mathrm{~d}$ postoperative morality rates $(\mathrm{p}<0.01)$. Perioperative hypothermia was associated with higher 48 $\mathrm{h}$ and $30 \mathrm{~d}$ postoperative mortality $(\mathrm{p}<0.001)$. Operations in which a perioperative adverse event was documented or which resulted in postoperative ICU admission also were associated with higher $48 \mathrm{~h}$ and $30 \mathrm{~d}$ postoperative mortality rates $(\mathrm{p}<0.0005)$. General anesthesia was associated with the highest mortality rate $48 \mathrm{~h}$ postoperatively $(\mathrm{p}<0.0005)$, but both MAC and general anesthesia were associated with high mortality rates $30 \mathrm{~d}$ postoperatively $(\mathrm{p}<0.0005)$. Mortality rates $48 \mathrm{~h}$ postoperatively did not vary by total time under anesthesia care. Mortality rates $30 \mathrm{~d}$ postoperatively did vary by total time under anesthesia care $(\mathrm{p}<0.01)$, but a positive linear relationship, in which mortality rates increased with increased anesthesia care time, was not apparent. The need for invasive monitoring via arterial line, cardiovascular catheter or pulmonary artery catheter was associated with higher mortality rates $48 \mathrm{~h}$ and $30 \mathrm{~d}$ postoperatively $(\mathrm{p}<0.002)$. Deliberate intraoperative hypotension was not associated with postoperative mortality.

The evaluated risk factors were combined as binary variables in a multivariate generalized estimating equations

Table I Hospital deaths after inpatient surgeries ${ }^{\mathrm{a}}$

Inpatient surgeries $n=12,739$

\begin{tabular}{ll}
\hline Death within $\mathbf{4 8}$ hours & $72(0.57 \%)$ \\
Incidence & $\mathrm{I}: 177$ \\
$\begin{array}{l}\text { Mortality rate } \\
\text { Death within } \mathbf{3 0} \text { days }\end{array}$ & $262(2.1 \%)$ \\
Incidence & $\mathrm{I}: 49$ \\
Mortality rate &
\end{tabular}

Notes: ${ }^{a}$ Over the sampling period of 4/I/04-3/3//05, I2,739 inpatient surgical procedures. The table lists the cumulative incidence (frequency and percent) of hospital deaths, and the overall mortality rate, over two time periods, 48 hours and 30 days after surgery. 
A.

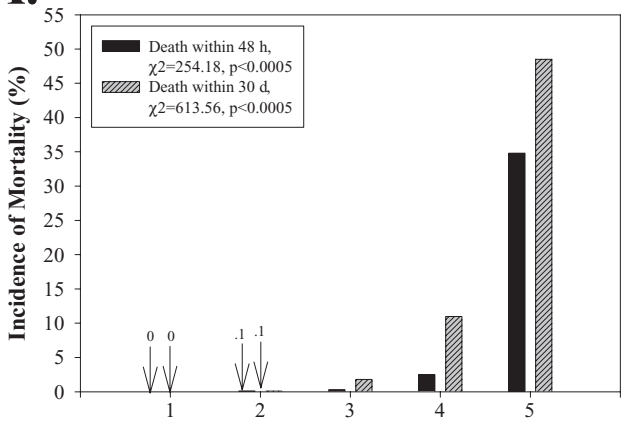

ASA Physical Status of Patient

C.

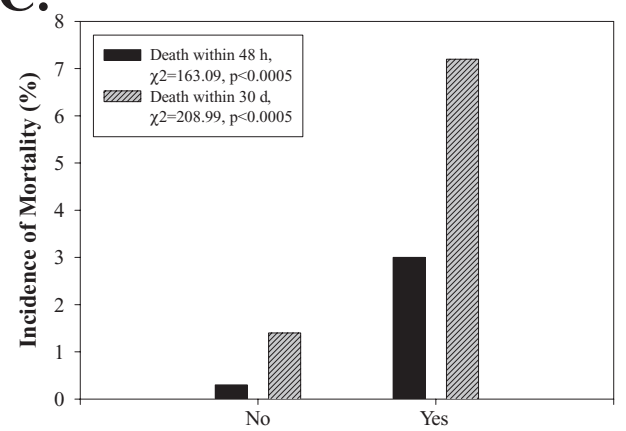

Emergency Procedure

E.

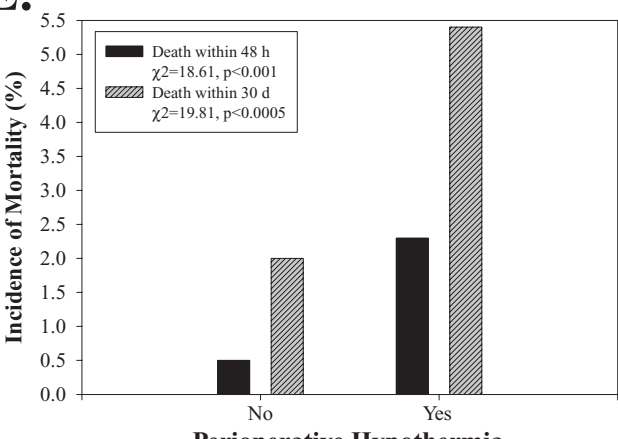

B.

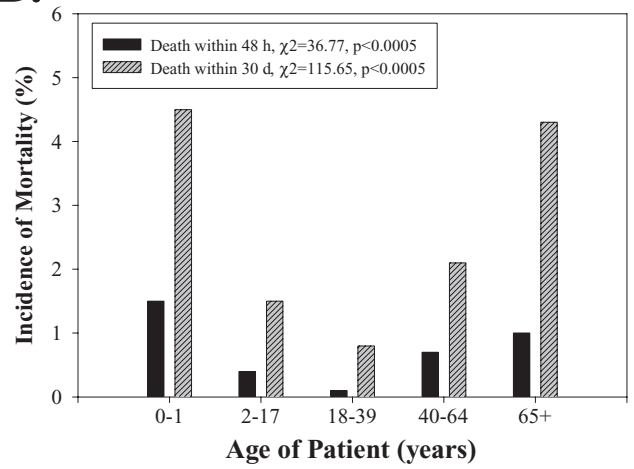

D.

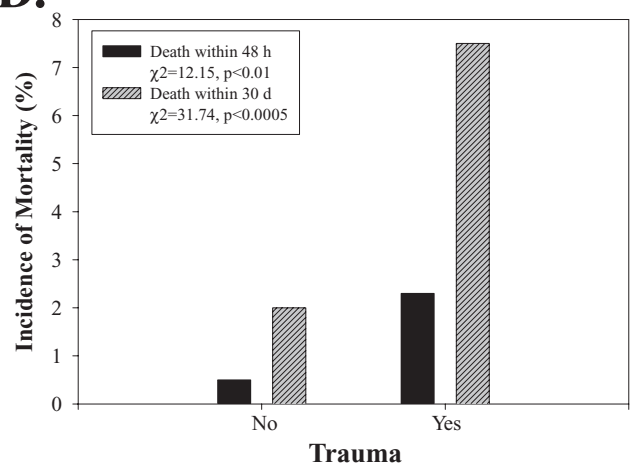

F.

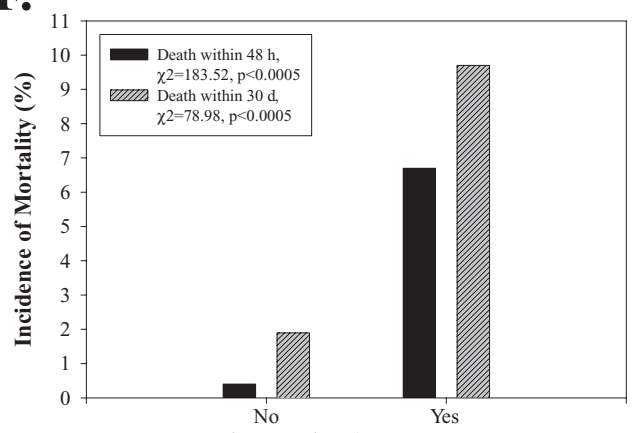

Perioperative Adverse Event

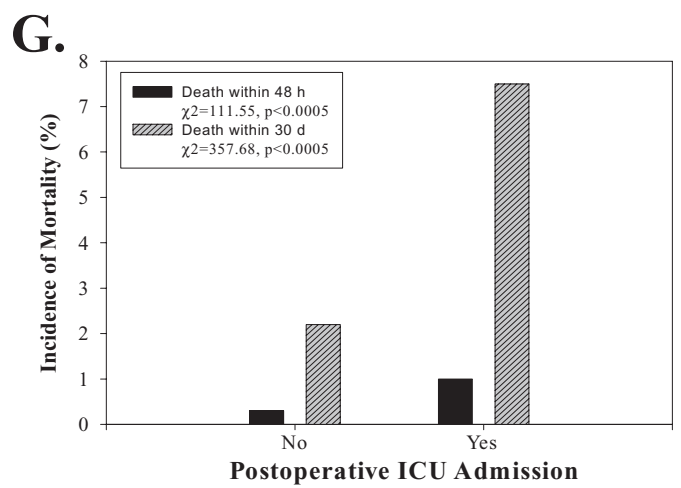

Figure I Risk factors for $48 \mathrm{~h}$ and $30 \mathrm{~d}$ postoperative mortality. For each panel, the $\mathrm{y}$-axis shows the incidence of mortality (\%) $48 \mathrm{~h}$ or $30 \mathrm{~d}$ postoperatively, and the $\mathrm{x}$-axis shows the ASA PS of the patient (A; I-5 scale), the patient's age (B; years), the emergency status of the operation (C), whether the operation was a trauma case (D), whether the patient was hypothermic perioperatively $(\mathbf{E})$, whether a perioperative adverse event occurred $(\mathbf{F})$, and whether the operation resulted in postoperative intensive care unit admission (G). In some panels, the exact percentage is noted with an arrow (eg, " 0 " refers to " $0 \%$ ").

Notes: The figure legends list the significance levels from chi-square or Fisher's exact test examining incidence rates across each level of the risk factor. Abbreviations: ASA PS, American Society of Anesthesiologists physical status; d, day; h, hour; NS, not significant. 
A

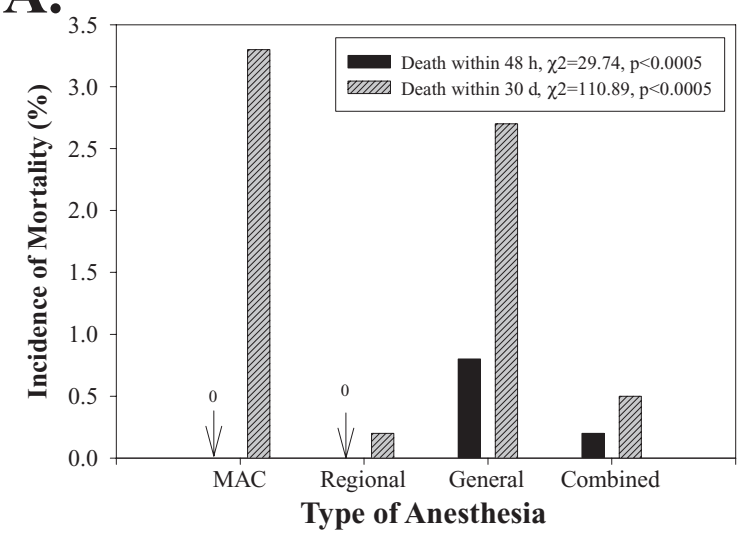

C.

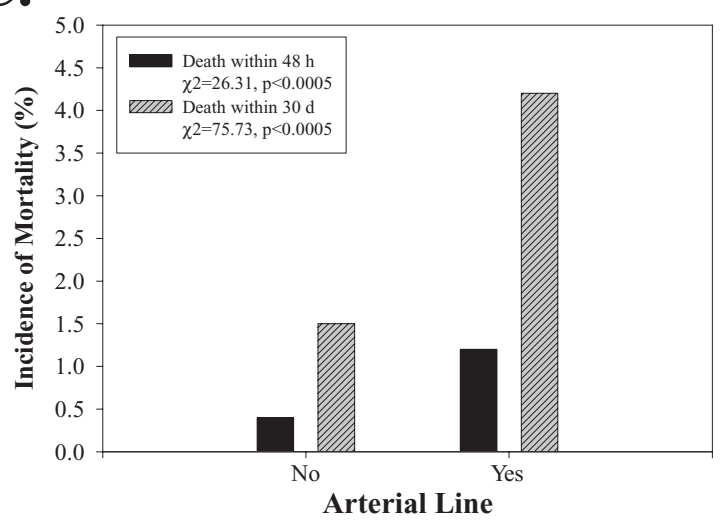

E.

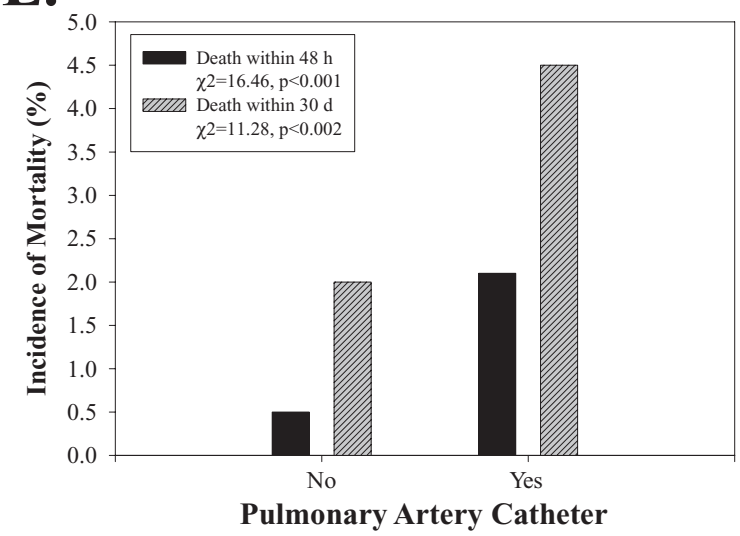

B.

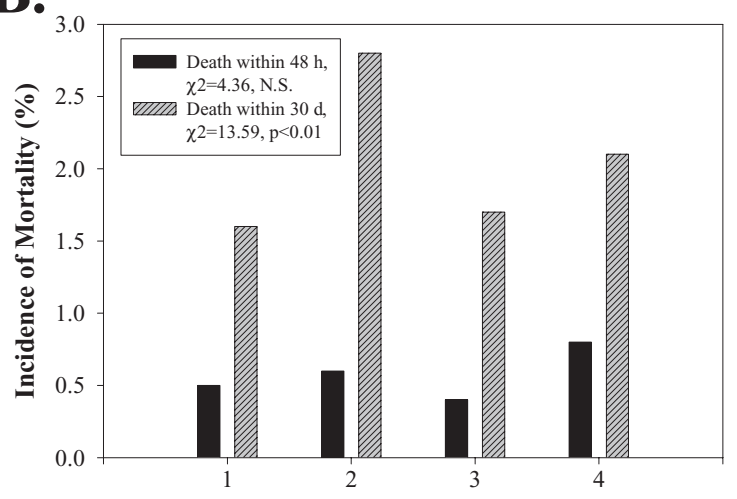

Total Time Under Anesthesia Care (quartiles)

D.

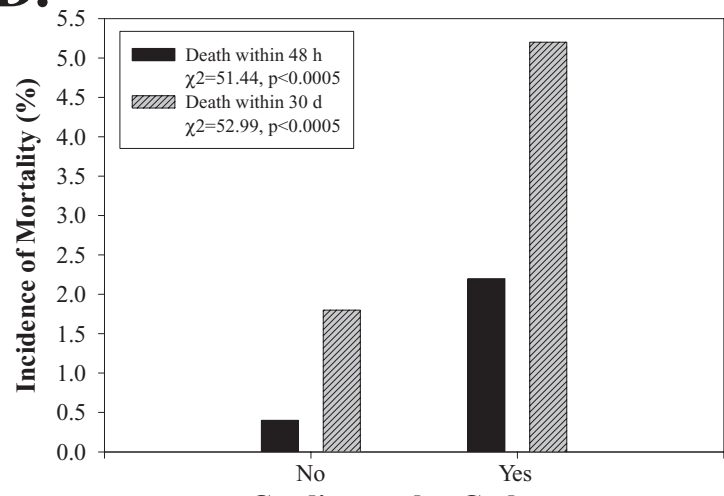

Cardiovascular Catheter

F.

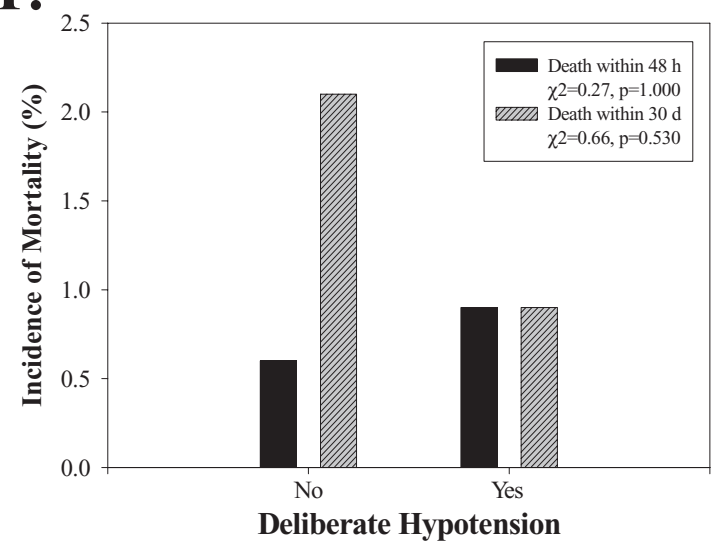

Figure 2 Risk factors for $48 \mathrm{~h}$ and $30 \mathrm{~d}$ postoperative mortality. For each panel, the $\mathrm{y}$-axis shows the incidence of mortality (\%) $48 \mathrm{~h}$ or $30 \mathrm{~d}$ postoperatively, and the $\mathrm{x}$-axis shows the anesthesia type (A; MAC, regional anesthesia, general anesthesia or combined), the total time under anesthesia care (B; minutes), the use of invasive monitoring via arterial line (C), cardiovascular catheter (D) or pulmonary artery catheter (E), and whether the operation involved deliberate intraoperative hypotension (F). In some panels, the exact percentage is noted with an arrow (eg, "0" refers to "0\%").

Notes: The figure legends list the significance levels from chi-square or Fisher's exact test examining incidence rates across each level of the risk factor. Abbreviations: $d$, day; h, hour; MAC, monitored anesthesia care; NS, not significant.

model to determine the strength of each factor as a predictor of postoperative mortality and to estimate odds ratios (Table 2). For $48 \mathrm{~h}$ postoperative mortality, ASA PS (3, 4, 5 vs. 1, 2), the occurrence of a perioperative adverse event, an emergency operation, postoperative ICU admission and the extremes of age (0-1 or $65+$ vs. 2-64) were identified as significant risk factors $(\mathrm{p}<0.05)$. The occurrence of a perioperative adverse event was the strongest risk factor, with an estimated odds ratio 
Table 2 Generalized estimating equations model for 48 hour and 30 day postoperative mortality

\begin{tabular}{|c|c|c|c|c|c|}
\hline & Coefficient (B) & Standard Error & $p$ value ${ }^{b}$ & $\operatorname{Exp}(B)^{c}$ & 95\% Cl for $\operatorname{Exp}(B)^{d}$ \\
\hline \multicolumn{6}{|l|}{48 hour postoperative mortality } \\
\hline ASA PS & 2.26 & 0.60 & $\mathrm{P}<0.0005$ & 9.57 & $2.97-30.85$ \\
\hline Age & 0.57 & 0.27 & $\mathrm{p}<0.05$ & 1.75 & $1.04-2.98$ \\
\hline Emergency Operation & 2.06 & 0.27 & $\mathrm{P}<0.0005$ & 7.81 & $4.57-13.34$ \\
\hline Trauma & 0.19 & 0.53 & N.S. & 1.21 & $0.43-3.43$ \\
\hline Perioperative Hypothermia & 0.30 & 0.47 & N.S. & 1.36 & $0.54-3.43$ \\
\hline Perioperative Adverse Event & 2.48 & 0.31 & $\mathrm{P}<0.0005$ & 11.91 & $6.46-21.96$ \\
\hline Postoperative ICU Admission & 0.88 & 0.31 & $\mathrm{p}<0.01$ & 2.42 & $1.32-4.43$ \\
\hline Total time under Anesthesia Care & 0.52 & 0.37 & N.S. & 1.67 & $0.81-3.45$ \\
\hline Anesthesia type & 1.06 & 0.71 & N.S. & 2.90 & $0.72-11.75$ \\
\hline Deliberate Hypotension & 0.49 & 1.28 & N.S. & 1.64 & $0.13-20.09$ \\
\hline Arterial Line & -0.47 & 0.37 & N.S. & 0.62 & $0.30-1.28$ \\
\hline Cardiovascular Catheter & 0.66 & 0.43 & N.S. & 1.93 & $0.83-4.49$ \\
\hline Pulmonary artery Catheter & -0.57 & 0.45 & N.S. & 0.56 & $0.23-1.37$ \\
\hline \multicolumn{6}{|l|}{30 day postoperative mortality } \\
\hline ASA PS & 2.72 & 0.37 & $\mathrm{P}<0.0005$ & 15.20 & $7.31-31.56$ \\
\hline Age & 0.59 & 0.14 & $\mathrm{P}<0.0005$ & 1.79 & $1.37-2.35$ \\
\hline Emergency Operation & 1.18 & 0.15 & $\mathrm{p}<0.0005$ & 3.25 & $2.42-4.37$ \\
\hline Trauma & 0.36 & 0.31 & N.S. & 1.43 & $0.78-2.61$ \\
\hline Perioperative Hypothermia & 0.15 & 0.29 & N.S. & 1.17 & $0.66-2.06$ \\
\hline Perioperative Adverse Event & 1.37 & 0.25 & $\mathrm{P}<0.0005$ & 3.92 & $2.4 I-6.37$ \\
\hline Postoperative ICU Admission & 1.09 & 0.15 & $\mathrm{P}<0.0005$ & 2.96 & $2.19-4.00$ \\
\hline Total Time Under Anesthesia Care & -0.08 & 0.19 & N.S. & 0.93 & $0.64-1.36$ \\
\hline Anesthesia type & 1.20 & 0.39 & $\mathrm{P}<0.01$ & 3.32 & $1.54-7.16$ \\
\hline Deliberate Hypotension & -0.68 & 1.06 & N.S. & 0.50 & $0.06-4.03$ \\
\hline Arterial line & -0.02 & 0.18 & N.S. & 0.98 & $0.69-1.39$ \\
\hline Cardiovascular Catheter & 0.08 & 0.25 & N.S. & 1.09 & $0.67-1.77$ \\
\hline Pulmonary artery Catheter & -0.66 & 0.30 & $\mathrm{P}<0.05$ & 0.52 & $0.29-0.93$ \\
\hline
\end{tabular}

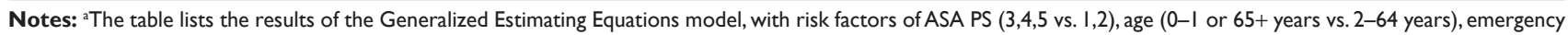
procedure (yes vs. no), trauma (yes vs. no), preoperative hypothermia (yes vs. no), perioperative adverse event (yes vs. no), ICU admission postoperatively (yes vs. no), total time under anesthesia care (top $25 \%$ vs. bottom $75 \%$ ), anesthesia type (general vs. MAC, regional or combined for death within $48 \mathrm{~h}$; general or MAC vs. regional or combined for death within 30 days), and invasive monitoring via arterial line, cardiovascular catheter or pulmonary artery catheter (yes vs. no); '“N.S. stands for that significant.”; 'Exp(B) is an estimate of the odds ratio for each risk factor; ${ }^{9} 95 \%$ Confidence Intervals $(\mathrm{Cl})$ are provided for each Exp(B) estimate.

Abbreviations: ASA PS, American Society of Anesthesiologists physical status; d, day; h, hour; ICU, intensive care unit; MAC, monitored anesthesia care; NS, not significant.

of 11.91 (95\% CI: 6.46-21.96; $\mathrm{p}<0.0005)$. The extremes of age (0-1 or 65+ vs. 2-64) was the weakest risk factor, with an estimated odds ratio of 1.75 (95\% CI: 1.04-2.98; $\mathrm{p}<0.05)$. For $30 \mathrm{~d}$ postoperative mortality, ASA PS (3, 4, 5 vs. 1, 2), anesthesia type (MAC or general vs. regional or combined), an emergency operation, the occurrence of a perioperative adverse event, postoperative ICU admission and the extremes of age (0-1 or $65+$ vs. $0-64)$ were identified as significant risk factors ( $\mathrm{p}<0.01)$. ASA PS $(3,4,5$ vs. 1,2$)$ was the strongest risk factor, with an estimated odds ratio of 15.20 (95\% CI: $7.31-31.56 ; \mathrm{p}<0.0005)$ and the extremes of age $(0-1$ or $65+$ vs. 2-64) was again the weakest risk factor, with an estimated odds ratio of 1.79 (95\% CI: 1.37-2.35; $\mathrm{p}<0.0005$ ). For 30 day postoperative mortality, the use of a pulmonary artery catheter for invasive monitoring was associated with a small decreased risk of mortality, with an estimated odds ratio of 0.52 (95\% CI: $0.29-0.93 ; \mathrm{p}<0.05$ ).

\section{Discussion}

Despite recent mandates for improvements in medication safety and adverse event tracking by the IOM, JCAHO, and IHI (IOM, 1999; 2001; IHI 2007; JC 2007), surprisingly little recent research exists on anesthesia safety in the United States, either in terms of tracking adverse event rates, identifying antecedents and risk factors, testing preventative interventions, or comparing rates of adverse events across institutions. Postoperative mortality is an objective endpoint that can be used to evaluate anesthesia safety, within and between institutions, and to test the effectiveness of changes in practice (Baker et al 2007). Our findings suggest that $48 \mathrm{~h}$ postoperative mortality at our hospital occurs at a rate of 5.7 per 1000 operations $(0.57 \%)$. Lagasse (2002) recently conducted a review of all studies on perioperative mortality published worldwide over the last five decades and found an overall perioperative mortality 
rate of approximately 2 per 1000 anesthetics (or $0.2 \%$ ). Our rates are higher than those approximated by Lagasse, but within the same range. Our findings further suggest that $30 \mathrm{~d}$ postoperative mortality at our hospital occurs at a rate of 21 per 1000 operations (2.1\%).

Postoperative mortality is associated with three major categories of risk factors: patient comorbidity; the surgical procedure itself; and risks directly related to anesthesia management (Beecher and Todd 1954). A major goal of our study was to begin to identify specific risk factors for postoperative mortality, and we carefully selected different variables that reflect each of these major risk categories. Patient comorbidity was captured in our statistical analysis by including subject identifier as a covariate in the generalized estimating equations model to control for subjects having multiple operations over our one year sampling period, as they are presumably sicker than other subjects. Several other surrogates of patient comorbidity also were directly evaluated. The ASA PS is a well established surrogate measure of patient comborbidity (Vacanti et al 1970; Tiret et al 1986; Schwilk et al 1989; Forrest et al 1992). Our analysis confirmed that higher ASA PS scores are associated with increased risk of both $48 \mathrm{~h}$ and $30 \mathrm{~d}$ postoperative mortality. Notably, no patients classified as ASA PS 1 died $48 \mathrm{~h}$ or $30 \mathrm{~d}$ postoperatively and very few patients classified as ASA PS 2 died over the two postoperative time periods. However, approximately $35 \%$ of ASA PS V patients died within 48 $\mathrm{h}$ and nearly $50 \%$ of those patients died within $30 \mathrm{~d}$ postoperatively. Both $48 \mathrm{~h}$ and $30 \mathrm{~d}$ postoperative mortality rates were higher after emergency operations or after operations resulting in postoperative ICU admission. The odds ratio for death after an emergency procedure was greatest for the first $48 \mathrm{~h}$ after inpatient surgery, but was significant after $30 \mathrm{~d}$ as well, which is somewhat surprising. An emergency procedure imparted approximately 8 times increased risk of death within $48 \mathrm{~h}$ and 3 times increased risk of death within $30 \mathrm{~d}$ postoperatively. Postoperative ICU admission was associated with a 2-3 times increased risk of $48 \mathrm{~h}$ or $30 \mathrm{~d}$ postoperative mortality. Trauma and perioperative hypothermia were not identified as risk factors for postoperative mortality in our multivariate analysis.

The need for invasive monitoring, via arterial line, cardiovascular catheter or pulmonary artery catheter, reflects an increase in patient comorbidity and/or surgical complexity. Our multivariate analysis showed that invasive monitoring was not associated with increased risk of $48 \mathrm{~h}$ or $30 \mathrm{~d}$ postoperative mortality. Total time under anesthesia care is considered another surrogate measure of surgical complexity.
Indeed, complex operations take longer to complete and are associated with greater physiological stress and trauma for the patient. Early studies by other groups demonstrated a relationship between longer time under anesthesia care and greater risk of any type of perioperative adverse event (Beecher and Todd 1954; Tiret et al 1986; Schwilk et al 1998). Our study did not find an association between time under anesthesia care and postoperative mortality. Deliberate intraoperative hypotension is sometimes required during complex operations. Our analysis also did not find an association between deliberate intraoperative hypotension and postoperative mortality. Collectively, these findings suggest that anesthesiologists are managing longer, more complex operations quite well.

The occurrence of an adverse event in the immediate perioperative period was associated with higher $48 \mathrm{~h}$ and $30 \mathrm{~d}$ postoperative mortality. A surgery associated with a perioperative adverse event imparted a 12 times increased risk of death within $48 \mathrm{~h}$ postoperatively and 4 times increased risk of death within $30 \mathrm{~d}$ postoperatively. Although we did not analyze subcategories of perioperative adverse events, cardiovascular and respiratory events were the most common. However, we cannot determine from the current data whether these events reflect patient comorbidity, anesthetic complications, or surgical complexity. Further studies, using a larger sample and greater resolution data, will explore this relationship and investigate the specific contribution of different perioperative adverse events to postoperative mortality.

Earlier studies identified the extremes of age as a risk factor for perioperative adverse events (Beecher and Todd 1954; Eger et al 1971; Farrow et al 1982; Tiret et al 1986; Forrest et al 1992; Schwilk et al 1998). In our study, infants (0-1 years) and older persons (65+ years) experienced higher rates of postoperative mortality than persons 2-64 years. However, the extremes of age imparted less than 2 times increased risk of $48 \mathrm{~h}$ or $30 \mathrm{~d}$ postoperative mortality. These findings suggest that patients in the extreme age groups are now being managed relatively well.

Whether general anesthesia is associated with greater risk than regional anesthesia, or vice-versa, is a matter of great debate (Gulur et al 2006). Our study did not fully clarify the relationship between postoperative mortality and the type of anesthesia used during surgery. Anesthesia type was not identified as a significant risk factor for $48 \mathrm{~h}$ postoperative mortality, but for $30 \mathrm{~d}$ postoperative mortality, the use of MAC or general anesthesia versus regional or combined anesthesia was identified as a significant risk factor. The identification of MAC as a significant risk factor might 
reflect the anesthesiologist's decision to use MAC instead of general anesthesia for sicker patients, and so the significant association might reflect a relationship between patient comorbidity, not anesthesia type, and $30 \mathrm{~d}$ postoperative mortality. Our results add to the debate in the literature regarding the risks associated with regional versus general anesthesia, but further studies clearly are needed.

\section{Limitations}

Our study has several limitations that should be considered when interpreting the results. First, this was an observational study and as such, it is vulnerable to both confounding and misclassification bias. Second, our mortality data are derived solely from our hospital's Surgical Services database, and so our data do not reflect deaths occurring outside of our hospital. Third, the relationship between the identified risk factors and death is not necessarily causal, especially for the $30 \mathrm{~d}$ period, in which multiple additional variables which we were unable to capture are likely to be involved.

\section{Implications}

Our results indicate that postoperative mortality occurs at high rates. Our study identified new and re-established other risk factors for postoperative mortality in the modern operating room environment, and our study discounted several risk factors identified in early studies. We aim to build upon our findings, track mortality rates at our institution and across others, and test interventions designed to reduce postoperative mortality rates and increase anesthesia safety.

\section{Acknowledgments}

The authors gratefully acknowledge the mentorship provided by Dr. Robert A Mueller, M.D., Ph.D., and the contributions of all current and former CRNAs, residents, fellows, and attendings affiliated with our Department of Anesthesiology.

\section{References}

Baker R, Sullivan E, Camosso-Stefinovic J, et al. 2007. Making use of mortality data to improve quality and safety in general practice: A review of current approaches. Qual Saf Health Care, 16:84-9.

Beecher HK, Todd DP. 1954. A study of the deaths associated with anesthesia and surgery: Based on a study of 599,548 anesthesias in ten institutions 1948-1952, inclusive. Ann Surg, 140:2-35.
Cooper JB, Cullen DJ, Nemeskal R, et al. 1987. Effects of information feedback and pulse oximetry on the incidence of anesthesia complications. Anesthesiology, 67:686-94.

Cooper J, Gaba, D. 2002. No myth: Anesthesia is a model for addressing patient safety. Anesthesiology, 97:1335-7.

Dripps RD, Lamont A, Eckenhoff JE. 1961. The role of anesthesia in surgical mortality. JAMA, 178:261-6.

Eger EI 2nd, Bahlman SH, Munson ES. 1971. The effect of age on the rate of increase of alveolar anesthetic concentration. Anesthesiology, $35: 365-72$.

Eichhorn JH. 1989. Prevention of intraoperative anesthesia accidents and related severe injury through safety monitoring. Anesthesiology, 70:572-7.

Farrow SC, Fowkes FG, Lunn JN, et al. 1982. Epidemiology in anaesthesia. II: Factors affecting mortality in hospital. Br J Anesthesiol, 54:811-17.

Fecho K, Moore CG, Lunney AT, et al. In press. Perioperative adverse events: A comparison of inpatient and outpatient procedures. Intl J For Health Care Qual Assur, in press.

Forrest JB, Rehder K, Cahalan MK, et al. 1992. Multicenter study of general anesthesia. III. Predictors of severe perioperative adverse outcomes. Anesthesiology, 76:3-15.

Gulur P, Nishimori M, Ballantyne JC. 2006. Regional anaesthesia versus general anaesthesia, morbidity and mortality. Best Pract Res Clin Anaesthesiol, 20:249-63.

[IHI] Institute for Healthcare Improvement. 2007. Status quon't - Why health care will never be the same [online]. Accessed May 16, 2007. URL: http://www.ihi.org.

[IOM] Institute of Medicine. 2001. Crossing the quality chasm: A New Health System for the 21st Century [online]. Accessed May 16, 2007. URL: http://www.nap.edu/books/0309072808/html.

[IOM] Institute of Medicine, Committee on Quality of Health Care in America. 1999. To err is human: Building a safer health system. Washington, DC: Natl Acad Pr.

Lagasse RS. 2002a. Anesthesia safety: Model or myth? Anesthesiol, 97:1609-17.

Lagasse RS. 2002b. Indicators of anesthesia safety and quality. Curr Opin in Anaesthiol, 15:239-43.

Monk TG, Saini V, Weldon BC, et al. Anesthetic management and one-year mortality after noncardiac surgery. Anesth Analg, 100:4-10.

[JC] The Joint Commission. 2007. Improving America's Hospitals: A Report on Quality and Safety [online]. Accessed May 16, 2007. URL: http://www.jointcommissionreport.org.

Schwilk B, Muche R, Treiber H, et al. 1998. A cross-validated multifactorial index of perioperative risks in adults undergoing anaesthesia for noncardiac surgery. J Clin Monit Comp, 14:283-94.

Tiret L, Desmonts JM, Hatton F, et al. 1986. Complications associated with anaesthesia - A prospective survey in France. Can J Anesth 1986; 33:336-44.

Vacanti CJ, VanHouten, RJ, Hill RC. 1970. A statistical analysis of the relationship of physical status to postoperative mortality in 68,388 cases. Anesth Analg, 49:564-6.

Warner MA, Shields SE, Chute CG. 1993. Major morbidity and mortality within 1 month of ambulatory surgery and anesthesia. JAMA, 270:1437-41. 